\title{
col|aboratory
}

III INDIANA UNIVERSITY

\section{Concept Paper \\ Re-Imagining Benchmarking as Stewards of Place: Identifying Peers in the Context of Communities}

Kristin Medlin, MPA, MS

Kristin Norris, PhD

Emily M. Janke, PhD

July 2021

Please cite this document as:

Medlin, K., Norris, K., and Janke, E. (2021). Re-Imagining Benchmarking as Stewards of Place: Identifying

Peers in the Context of Communities (Version 1.0). 
Introduction

As Stewards of Place, it is essential that our institutions engage in strong campus-community partnerships, perhaps now more than ever, to leverage the resources and knowledge to address pressing community-identified issues. ${ }^{1}$ How can we ensure that we create more equitable and responsive relationships within our communities and with our community partners? How can our institutions of higher education learn not just from each other, but from the communities in which they are situated? Higher education needs to better understand engagement in this new landscape in order to build more intentional, mutually beneficial, and equitable partnerships. This requires institutions to define the who, what, where, when and why of strategies, activities, and programming with and for our communities. During times of financial uncertainty and societal unrest, how do we leverage data about our campus-community partnerships to achieve common goals? To foster dialogue? To examine impacts and resources leveraged? How do we tell a robust story of engagement?

\section{Benchmarking: A Primer}

To answer these questions, we return to the roots of benchmarking in higher education. Benchmarking gained popularity in higher education in the 1990s when institutions were increasingly called to demonstrate effectiveness according to standardized measures. ${ }^{2}$ This was an era of continuous quality improvement, during which institutions identified the quality of an organization or program based on a select set of common indicators. By creating standardized measures that were commonly used across institutions, institutional leaders could not only set goals and measure their progress towards them, but also set and measure themselves in relation to others. Knowing one's peers and aspirational peers allowed institutional leaders to identify others from which they could learn about strategies and best practices as well as distinguish themselves through comparisons, particularly if a peer is seen as prestigious.

However, as is the case with many efforts to establish common measures, the measures became the end in and of themselves. Levy and Ronco (2012) suggested that:

with the rush for greater accountability, the thoughtful processes envisioned by the concept of benchmarking were quickly replaced with a lust for quick measurement and data. As attractive as this option may be, the sole use of performance indicators ignores a basic and powerful premise of benchmarking-namely, that what is needed for

\footnotetext{
${ }^{1}$ American Association of State Colleges and Universities (2002). Stepping Forward as Stewards of Place: A Guide for Leading Public Engagement at State Colleges and Universities. Washington, DC: AASCU.

${ }^{2}$ Levy, G. D., \& Ronco, S. L. (2012). How benchmarking and higher education came together. New Directions for Institutional Research, 2012(156), 5-13. https://doi.org/10.1002/ir.20026
} 
improvement is to identify the processes behind the benchmarks or metrics that can lead to improved performance, subsequently demonstrated by associated benchmarks or metrics.

More colorfully, Birnbaum (2001) asserted that what ultimately found a home in higher education was not benchmarking at all, but a "half-sibling called performance indicators and a kissing cousin called performance funding." ${ }^{3}$ Institutional leaders no longer viewed benchmarking as a strategy to improve quality and effectiveness by identifying and sharing best practices among identified peer institutions, but rather to rank institutions against each other as image management.

\section{Placemarking: Beyond Benchmarking Theory}

We have coined the term "Placemarking" as a play on words. It emphasizes not the "bench," or the measure, but rather the "place" in which the institution resides and is inextricably embedded. Whereas traditional benchmarking approaches require measures related to institutional characteristics (e.g., public/private, undergraduate/graduate), and to some extent, community characteristics (e.g., rural/urban, state), Placemarking emphasizes sociological data about the community and requires data collected by institutions about their community-campus partnerships. The purpose of Placemarking is to help institutions understand, advance, and align institutional and community priorities.

Placemarking enhances benchmarking by including the community context in which institutions reside and how institutions work in partnership with their communities (e.g., its people, organizations, issues, and assets). Placemarking allows institutions to better align their community-oriented identity and image, advocate on behalf of both institutional and community priorities, and fosters collaborative planning.

Placemarking metrics include:

- Institutional characteristics: student characteristics, student finances, faculty and expenses, revenue sources, and degrees (i.e., IPEDS, Carnegie Classification for the Advancement of Teaching)

- Community characteristics: demographic, organizational, and community characteristics (i.e., Opportunity Indexes - economy, education, health and community indicators)

- Community-university partnerships: standardized data describing partnership work (i.e., community engagement and public service data collected via Collaboratory)

\footnotetext{
${ }^{3}$ Birnbaum, R. Management Fads in Higher Education. San Francisco: Jossey-Bass, 2001.
} 
This white paper shares an introduction to our Placemarking theory and discusses the potential of examining partnerships using Placemarking. Future publications will introduce examples of how the Model can be leveraged to identify "engaged peers."

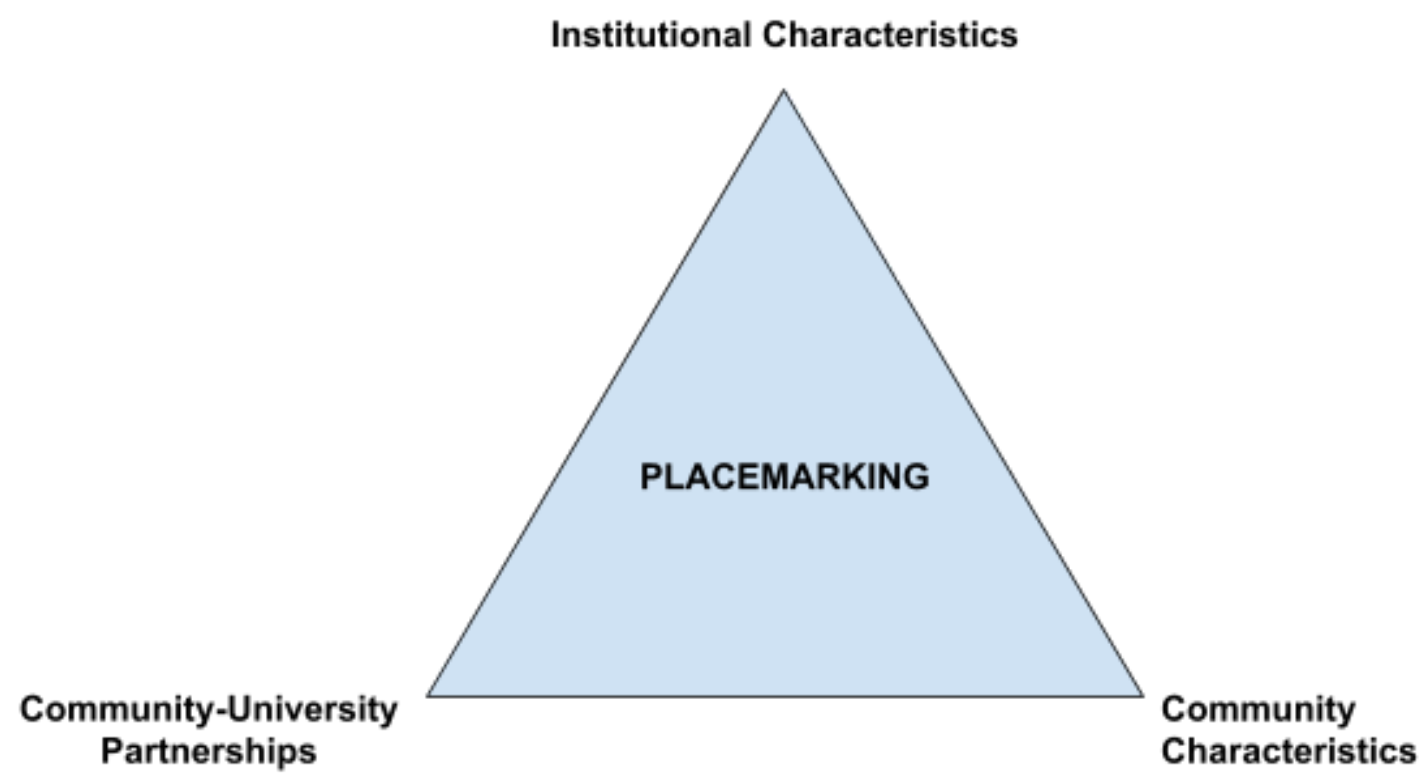

Figure 1. Placemarking Conceptual Model

\section{Institutional Characteristics}

To understand institutional characteristics, most institutions in the United States report data through the Integrated Postsecondary Education Data System, or IPEDS. ${ }^{4}$ IPEDS "provides basic data needed to describe - and analyze trends in - postsecondary education, in terms of the numbers of students enrolled, staff employed, dollars expended, and degrees earned." Conducted each year by the U.S. Department of Education's National Center for Education Statistics, IPEDS is a system of interrelated surveys that gathers information from every college, university, and technical and vocational institution that participates in the federal student financial aid programs as required by the Higher Education Act of 1965. IPEDS data are typically completed by Institutional Research offices. Examples of such data include:

- Enrollments

- Program completions

- Graduation rates

- Faculty and staff

- Finances

- Institutional prices

- Student financial aid

\footnotetext{
${ }^{4}$ U.S. Department of Education, National Center for Education Statistics, Integrated Postsecondary Education Data System (IPEDS). Retrieved from https://nces.ed.gov/ipeds/datacenter/InstitutionByName.aspx?goToReportld=1 on June 15, 2021.
} 
Beyond IPEDS, many institutions also join professional associations that support higher education community engagement (e.g., Campus Compact, the International Association for Research on Service-Learning and Community Engagement, Imagining America). These memberships can be used as institutional indicators of support for engagement. The Carnegie Foundation also hosts an Elective Classification for Community Engagement, a recognition awarded to institutions who complete an extensive self-study in the institutionalization of community engagement. ${ }^{5}$ Combining IPEDS data with memberships and recognitions is an example of an alternative method of identifying institutional peers.

\section{Community Characteristics}

Placemarking encourages institutions to look beyond themselves for understanding best methods, processes, and practices. Institutions of higher education do not operate in isolation, but instead, are part of an ecosystem within their community.

To examine this aspect of Placemarking, we look at the socioeconomic and demographic indicators for the communities within which institutions are located. We prioritize nationally available datasets with some level of granularity/comprehensiveness (e.g., data at the county, zip code, or census tract level, across all characteristics, across the United States). To date, the best set of indicators to serve our purpose is the Opportunity Index, developed and supported by Opportunity Nation, a campaign of the Forum for Youth Investment and Child Trends. ${ }^{6}$

The Opportunity Index provides a series of indicators that demonstrate what opportunity looks like in the United States across four dimensions of community well-being:

- Economy

- Education

- Health

- Community

The Index provides Opportunity Scores for all 50 states plus the District of Columbia and 2,065 counties, which together represent 97 percent of the US population. For the nation, the 2019 Opportunity Score stands at 53.2 out of 100. Currently, we are looking at the following indicators in support of our Placemarking Model:

- Economy

$$
\begin{array}{ll}
\circ & \text { Jobs } \\
\circ & \text { Wages } \\
\circ & \text { Poverty }
\end{array}
$$

\footnotetext{
${ }^{5}$ Carnegie Foundation for the Advancement of Teaching. (2011). Classification description: Community engagement elective classification.

${ }^{6}$ Opportunity Nation (2019). Opportunity Index [Data set]. https://opportunityindex.org/
} 
- Income Inequality

- Access to Banking Services

- Affordable Housing

- Broadband Internet Subscription

- Education
- Preschool Enrollment
- High School Graduation
- Postsecondary Education

- Community

○ Volunteering

- Voter Registration

- Youth Disconnection

- Violent Crime

- Access to Primary Healthcare

- Access to Health Food

- Incarceration

- Health

- Low Birth Weight

- Health Insurance Coverage

- Deaths Related to Alcohol/Drug Use and Suicide

\section{Community-University Partnerships}

While the first two elements of Placemarking look at institutional and community characteristics separately, the third element of "Institutional Activity" represents community-institutional interactions in order to analyze collective contributions to the community. To measure this, we look at data from Collaboratory ${ }^{7}$, a standardized nation-wide platform used by colleges and universities to track community engagement and public service activities. The Collaboratory dataset includes descriptive characteristics (what, where, when, with whom, and to what end) of activities that are entered by faculty and staff or their representatives.

While community and institutional data serve as helpful tools for identifying like "peers", Collaboratory data serves as the lens through which we can examine the activities of those peers. For example, does engagement look different for urban-serving institutions that have a lower Opportunity Index Score? Do they work with similar types of partners or funders, and do they operate similar programs?

\footnotetext{
${ }^{7}$ Collaboratory (Version 2.1.1). [Software]. HandsOn Connect Cloud Solutions (2021). Available from www.cecollaboratory.com.
} 
Collaboratory provides a highly relational view of the focus areas addressed, populations served, on- and off-campus collaborators, connections to teaching and research, and location information, among other data points. For example:

- Focus Areas

- Arts and Culture (e.g., local history, literary arts, cultural appreciation/celebration, dance, design, public arts)

- Community and Economic Development (e.g., business development, career and professional development, entrepreneurship, financial literacy, international/global business, labor relations, program evaluation, strategic planning, technology/web assistance, workforce development)

- Education (e.g., access for underrepresented populations, adult/lifelong learning, dropout prevention, English as a second language, literacy, STEM, school readiness, mentoring, professional development for teachers, information technology and computer literacy, K-12 education)

- Environmental Sustainability (e.g., brownfields, climate literacy, environmental education, food advocacy, land use planning, sustainability, water quality, energy, science literacy of the public)

- Government and Public Safety (e.g., child advocacy, citizenship, crime prevention, public policy analysis, re-entry programs)

- Health and Wellness (e.g., addiction treatment/prevention, health disparities/access, mental health, nutrition, parks and recreation, physical disabilities, sexual health)

- Social Issues (e.g., at-risk youth, civil rights, cultural understanding, disability issues, DEI, homelessness, hunger, immigration issues, poverty, PBGTQ issues, religious understanding, voting registration)

- Target Populations (e.g., African Americans, immigrants, families, urban communities, general public, Hispanic, Latino, or Spanish origin, indigenous, adults, infants, children, youth, young adults)

- Community Partners

- Type of organization (e.g., government, non-profit, k-12 education, for-profit)

- Physical address

- Role(s) of the community organization in the partnership (e.g., co-investigator, discuss how to work together, manage the event or activity, provide access to target populations, provide funds for the activity, provide space, supervise students, identify expected measures, benefits, or outcomes, co-educate students)

- Location of the engagement activity (if applicable)

- Student involvement (e.g., course, student organization/group, volunteers, student employee, graduate assistant/fellowship)

- If connected to a course, additional information is collected about the course: 
- Course number, title, section number

- Number of students enrolled

- Number of hours students serve

- Type of High Impact Practices employed

- Learning Objectives

- Scholarship (e.g., methodology, IRB \#, scholarly outputs)

The collection and documentation of institutional activity (community engagement and public service) is traditionally spread across multiple reporting platforms. Reporting processes and platforms tend to be unique to each institution making comparisons across institutions difficult. However, Collaboratory standardizes the data collected across all institutions. Below are some examples of the types of data institutions may systematically collect relative to community engagement and public service:

- Courses - high-impact practices, community partner names and sector (if applicable), number of students, number of service hours

- Co-Curricular engagement - students organizations, volunteer sites, how many students served, number of service hours

- Faculty service in the community - name of organization, role, length of term

- Programs in the community - partners, faculty/staff involvement, student involvement

- Research (i.e., community-engaged research or public scholarship) - IRB, faculty outputs (e.g., publications, white papers)

The value of Collaboratory is its standardization of the data collected and the ability to leverage pieces of information from existing systems and processes on campus to capture a more robust description of the activity and the myriad ways in which people, units on campus, and community partners are connected to address a pressing community-identified problem. Because of this standardization, we are able to conceive Placemarking, a way to identify peers based not only on institutional and community data, but also on the actual community engagement of the campuses' faculty, staff, and students.

\section{Conclusions and Future Applications}

Placemarking is a useful approach for institutions of higher education that seek to understand, increase, and deepen their public purpose and contributions to the communities in which they are located. By identifying appropriate peers in a community-engaged context, versus one that is based only on traditional characteristics and stakeholders, institutions can look to other similar communities for insights into partnership best practices, strategic alignment, and program improvement. Understanding ones' institutional portrait as characterized by its 
community characteristics as well as engagement activity enables an asset-based approach ${ }^{8}$ to engagement and encourages cross-sector stakeholder participation.

Placemarking allows institutions to: ${ }^{9,10}$

- Understand the pressing social issues of the communities in which they reside

- Take on the obligations of anchor institutions to positively impact the communities in which they are members

- Understand the full range and scope of community and economic engagement purposes, activities, impact areas, and constituents

- Clarify goals for engagement as a teaching pedagogy, as well as approach to research, creative activities

- Craft plans to support activities based on best practices and informed by campus-level data

- Identify opportunities for cross-institutional collaboration and learning

- Collect immediately accessible and real stories to assist development, advancement, and recruitment offices in raising funds, applicants, and advocates

- Strengthen one's reputation as a proactive and responsive member of the greater community, locally and beyond.

Additionally, in an era in which colleges and universities are compelled to compete for students, faculty, staff, and resources, the ability to distinguish themselves amongst others is crucial. Placemarking data can help institutions better understand and market themselves, improve their programs, and attract and recruit prospective students, families, and employees. The strategic advantage of Placemarking is that anyone can compare and contrast institutions according to actual engagement with communities, as well as other community and institutional characteristics.

Placemarking allows prospective students, employees and advocates to explore:

- What is special about this institution?

- What opportunities are available and supported with regards to active engagement with various social issues?

- What are the characteristics of the community that provide the opportunity to allow one to engage meaningfully?

\footnotetext{
${ }^{8}$ Kretzmann, J.P., \& McKnight, J.L. (1993). Building communities from the inside out: A path toward finding and mobilizing a community's assets. Chicago, IL: ACTA Publications.

${ }^{9}$ Adapted from Janke, E.M., \& Holland, B.A. (2013). Data Matter. Retrieved from https://communityengagement.uncq.edu/wp-content/ uploads/2014/07/Data-Matters.pdf.

${ }_{10}$ Janke, E. M., \& Medlin, K. D. (2017). Aligning Community and University Strengths and Priorities. Excellence in Community Engagement and Community-Engaged Scholarship. (Vol. 3). University of North Carolina at Greensboro: Institute for Community and Economic Engagement.
} 
- What are the avenues through which students, faculty, and staff are involved in and with communities?

\section{Call to Action}

Institutions of higher education need to expand their self-assessment processes to include how they achieve their mission and goals through partnerships with those external to the campus. This includes how they measure student learning and success and how the institution's community context contributes to and enhances student outcomes. In today's tumultuous world, student development of civic-mindedness is more important than ever. Civic learning and democratic engagement has deep roots in the curriculum and co-curriculum, but has nuanced differences in it's implementation across campuses and amongst faculty and disciplines. Institutions of higher education must curate meaningful and purposeful interactions that lead to civic action and move the needle on community priorities. Placemarking holds the potential to enhance the assessment of student civic knowledge and actions; examining the contexts, relationships, and level of reciprocity within a community engaged activity may explain what students learn and under what circumstances student development of civic-mindedness and civic action occurs.

To maximize the potential of Placemarking, institutions must first start by systematically documenting their work in and with the community. While many institutions have developed their own systems and processes for doing this to meet their immediate needs, the true potential emerges when everyone is using the same data collection process and data points (i.e., Collaboratory). Systematic monitoring of community engagement and public service activities allows for standardization of descriptive data points across institutions, which in turn enables large-scale analysis.

The Placemarking model leverages this data standardization to facilitate alignment of institutional resources and strengths with community priorities. Placemarking re-imagines who constitutes a peer, allowing us to honor the roots of benchmarking for continuous improvement purposes. An institution's capacity to examine the strength of their partnerships and the extent to which they are building intentional and equitable partnerships is possible. Our goal is to apply the model to and illustrate key examples within the network of institutions of higher education currently using Collaboratory to track data. We hope to develop a methodology for identifying ideal community-engaged peer institutions and suggest that these will differ significantly from traditionally identified peers. 
Authors

\section{Kristin Medlin}

\section{kmedlin@cecollaboratory.com}

Kristin Medlin, MPA, MS oversees Collaboratory's research and development efforts that support, promote, and enhance the field of study related to higher education community engagement. She works to raise awareness about the value of community engagement data, the imperative to develop a data culture, and helps to build systems and structures for data awareness, access, analysis, and use. Prior to jumping into the corporate sector, Kristin served as the communications and partnerships manager in the Institute for Community \& Economic Engagement at UNC Greensboro where she co-founded Collaboratory alongside Drs. Emily Janke and Barbara Holland.

\section{Kristin Norris}

norriske@iu.edu

Kristin Norris, Ph.D. is the Associate Director for Indiana University's Office of School Partnerships, which bridges the gap between the IU campuses and P-12 classrooms to improve the quality and diversity of students enrolling in post-secondary education and career readiness. Kristin collaborates with faculty, staff and administrators on the IU campuses and with Hoosier educators, administrators and policy makers to build partnerships that improve k-12 education. Kristin is passionate about the public purpose of higher education in addressing community issues and how institutions leverage data about their community engagement to inform decision-making.

\section{Emily Janke}

\section{emjanke@uncg.edu}

Dr. Emily Janke is the founding director of the Institute for Community \& Economic Engagement and an associate professor of Peace \& Conflict Studies at UNC Greensboro. As a scholar-administrator she has advanced practice and scholarship in the areas of interdisciplinary and transdisciplinary scholarship with diverse teams, the equitable treatment of community-engaged scholarship in promotion and tenure policy and practices, strategic efforts to track and monitor community engagement and public service within and across institutions, and the use of restorative justice practices in interpersonal, intergroup, and institutional culture. Along with Barbara Holland and Kristin Medlin, Emily is an author of Collaboratory, a publicly searchable, online database that shares an institutional story about who, what, where, with whom, and to what ends community-university partners are working towards community-identified priorities for shared learning and mutual benefits. 